\title{
The Demonstration of a Light Extinction Tomography System at the NASA Glenn Research Center's Icing Research Tunnel
}

\author{
Mark R. Woike ${ }^{1}$, Timothy J. Bencic ${ }^{2}$, Jonathon D. Ponder ${ }^{3}$, Judith F. Van Zante ${ }^{4}$, \\ Laura King-Steen ${ }^{5}$, Emily N. Timko ${ }^{6}$ \\ NASA Glenn Research Center, Cleveland, Ohio, 44135 \\ David P. Rohler ${ }^{7}$ \\ Plexar Associates, Inc. Shaker Heights, OH, 44122 \\ Steven H. Izen ${ }^{8}$ \\ Case Western Reserve University, Cleveland, OH, 44106
}

\begin{abstract}
A prototype light extinction tomography system has been developed for acquiring realtime in-situ icing cloud uniformity and density measurements in the NASA Glenn Research Center's Icing Research Tunnel (IRT). These measurements are currently obtained manually during periodic cloud calibrations of the IRT. These manual measurements are time consuming and assume that cloud uniformity and density does not greatly vary between the periodic calibrations. It is envisioned that the new light extinction tomography system will provide the means to make these measurements in-situ in real-time and minimize the need for these manual measurements. This new system uses the principle of light extinction tomography to measure the spray density and distribution in the test section. The prototype system was installed and successfully demonstrated in the IRT in early 2018. Data sets were acquired for several typical spray and simulated fault conditions to assess system capability and sensitivity. This paper will describe the prototype light extinction system, the theory behind it, and the results of the demonstration test that was conducted in the IRT.
\end{abstract}

\section{Introduction}

The Icing Research Tunnel (IRT) creates conditions similar to an aircraft flying through clouds and is one of the most heavily used facilities at the NASA Glenn Research Center. The IRT differs from most wind tunnels in that the air can be cooled to temperatures well below freezing and it has a series of spray nozzles that produce super cooled water particles that simulate different types of clouds and icing conditions. These clouds of tiny water droplets are carried past a test article that is mounted in the $6 \mathrm{ft}$ high by $9 \mathrm{ft}$ wide test section. For consistent testing, this cloud should be as spatially uniform and repeatable as possible. Experimental protocol requires that the cloud be calibrated for uniformity and the total amount of water that flows through the test section.

\footnotetext{
${ }^{1}$ Research Engineer, Optics and Photonics Branch, 21000 Brookpark Rd, MS 77-1, AIAA Senior Member.

${ }^{2}$ Optical Diagnostics Expert, HX5 Sierra, 21000 Brookpark Rd, MS 6-2.

${ }^{3}$ Research Engineer, Optics and Photonics Branch, 21000 Brookpark Rd, MS77-1.

4 Technical Lead: Icing Engineering, Testing Division, 21000 Brookpark Rd, MS 6-7,AIAA Senior Member.

${ }^{5}$ Icing Cloud Calibration Engineer, HX5 Sierra, 21000 Brookpark Rd, MS 11-1.

${ }^{6}$ Icing Cloud Calibration Engineer, Jacobs Technology, 21000 Brookpark Rd, MS 11-1.

${ }^{7}$ President, 3120 Belvoir Rd.

${ }^{8}$ Professor of Mathematics, Consultant to Plexar Associates, Inc., 10900 Euclid Ave.
} 
The current method of acquiring icing cloud uniformity measurements is accomplished by bolting a metal grid in the test section and accreting ice on the exposed faces during an icing spray. Once the tunnel is operated at a test condition for a given spray, the facility is shut down and the accreted ice is manually measured by a technician entering the tunnel using calipers to measure the ice thickness to determine cloud uniformity. The cloud conditions are set by varying several different parameters including air speed, temperature, cloud water content and droplet size. Calibration is accomplished over the operational range of all of these parameters. Thus, the calibration coupled with the use of the current manual measurement techniques is very time consuming. A partial calibration is performed once every six months and a full calibration is performed approximately every 5 years.

A system based on light extinction tomography has been developed to aid in these calibrations and provide real-time monitoring of cloud uniformity and density between calibrations. This system monitors the spray distribution and density as a function of position in the test section. Sixty (60) sheet lasers spaced around the periphery of a $6 \mathrm{ft}$ by 9 $\mathrm{ft}$ measurement plane are sequentially projected across the test section, where one hundred twenty (120) light detectors, also spaced on the periphery, are used to measure the intensity of the light along the path from the lasers to the detectors. It takes two measurement conditions to generate an image of the density and uniformity of the cloud; a reference condition when no cloud is present, and a second when the cloud is present. The data sets are reconstructed using tomographic algorithms related to, but distinct from those used in medical imaging. A unique feature of this system is the rectangular geometry, which is very much different from the standard circular cross-section geometry encountered in most tomography applications. In addition, the reconstruction employs smooth basis functions as opposed to the sharper functions used in medical applications.

The prototype system developed in the lab was installed and demonstrated for the first time in the IRT in February of 2018. The prototype development apparatus was mounted slightly downstream of the $6 \mathrm{ft}$ by $9 \mathrm{ft}$ test section to save time and cost in not having to build a second system that would fit in the test section for this initial proof of concept demonstration. Data sets were acquired for a total of 79 standard spray and simulated fault conditions to determine the operability and sensitivity of the light extinction tomography system.

\section{Facility Description}

The IRT is a closed-loop refrigerated wind tunnel that simulates flight through an icing cloud. It is heavily used to study icing effects on aerodynamic vehicles and structures. A plan view of the facility is shown in Figure 1. A 5000 HP electric motor drives the $24 \mathrm{ft}$ fan made of wood from Sitka spruce. The fan drives air through expanding turning vanes in C-Corner, and into the face of the staggered heat exchanger. There, the air gets chilled/warmed within a temperature range of $20^{\circ} \mathrm{C}$ total to $-40{ }^{\circ} \mathrm{C}$ static. Twenty-four resistance temperature detectors (RTDs), distributed on the D-Corner contracting turning vanes, measure the total temperature in this plenum area. The heat exchanger is $26 \mathrm{ft}$ high and $50 \mathrm{ft}$ wide.

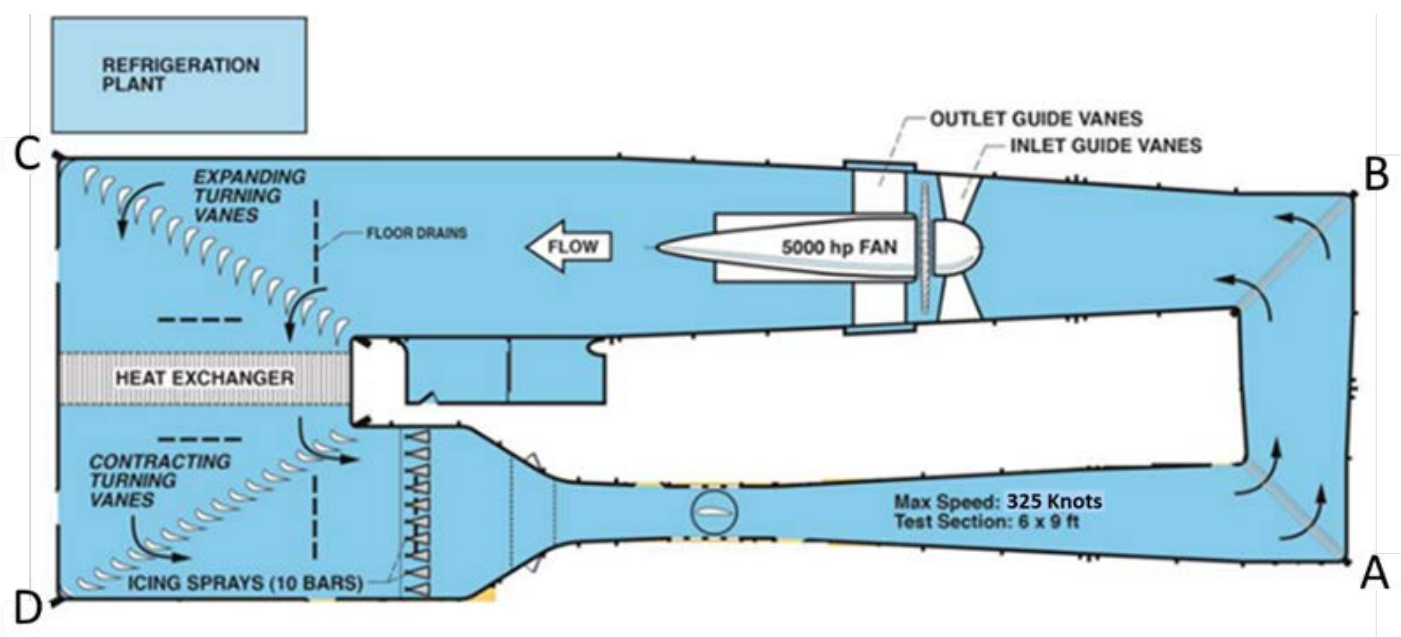

Figure 1.-Plan view schematic of the Icing Research Tunnel.

American Institute of Aeronautics and Astronautics 
The icing cloud conditions are generated downstream of D-Corner, where after the contracting turning vanes, there are 10 rows of spray bars with two different air-atomizing nozzle types: Mod1 (lower water flow rates) and Standard (higher water flow rates). Each bar has 55 nozzle positions; each position contains either a Mod1 nozzle, a Standard nozzle, or a plug. Each nozzle location is fed from two water manifolds through remotely controlled solenoid valves. It is possible to turn on only the Mod1 nozzles, only the Standard nozzles or both (with the same air pressure) to set various cloud conditions. The contraction area ratio into the test section is 14:1. The test section itself is $20 \mathrm{ft}$ long (axial) by $6 \mathrm{ft}$ high by $9 \mathrm{ft}$ wide. The center of the test section is $44 \mathrm{ft}$ from the spray bars. The calibrated speed range in the test section is from 50 to 325 knots. From the test section, the cloud continues to flow into the diffuser toward A-Corner, and on around into B-Corner and back into the fan.

Periodic calibrations are conducted to characterize the icing cloud conditions and assure uniformity. Cloud uniformity is measured using a 6 by $6 \mathrm{ft}$ grid in the test section. This grid extends floor to ceiling, and covers the central $6 \mathrm{ft}$ of the $9 \mathrm{ft}$ span. The grid mesh is 6 by 6 in. Mesh elements are 2 in. deep with a flat 1/8 in. face for ice accretion. During these calibrations, digital calipers are used to measure the ice thickness accreted at the center mesh points of the vertical elements. An image of a technician measuring ice on the grid during a typical calibration is shown in Figure 2. This manual method is time consuming and assumes that cloud uniformity and density does not greatly vary between the periodic calibrations.

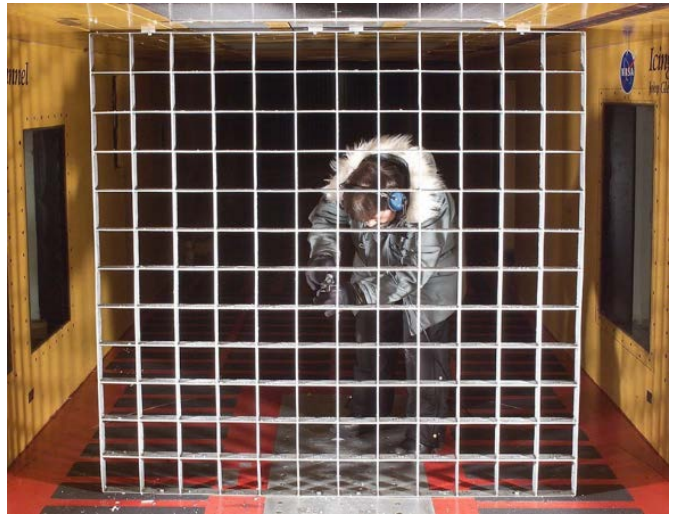

Figure 2.-Technician measuring the thickness of ice accreted on the Grid.

\section{Light Extinction Tomography System}

A prototype light extinction tomography system was developed to provide an in-situ measurement system to monitor a cloud in the test section of the IRT facility. While the initial system goal is to provide in-situ real-time monitoring of the cloud uniformity, the long term goal is to be able to make quantitative full-field water content measurements. To measure the low-spatial-frequency nature of super cooled water clouds, unique computed tomography (CT) algorithms have been developed that use Gaussian basis functions rather than pixel basis functions typically used in medical and structural analysis applications. Another feature of this system is that it utilizes a rectangular geometry as opposed to circular geometries used in typical applications. The system that was developed for the IRT is an extension of the work that was previously accomplished in implementing a circular cross-section geometry system for measuring icing spray conditions at the NASA Glenn Research Center’s Propulsion System Laboratory (PSL) ${ }^{1}$.

\section{A. Tomographic Algorithms for IRT Square Geometry}

Tomographic reconstruction is the recovery of a quantity from a collection of line integrals of the quantity. For this application, the relevant quantity is liquid water content. For the particle sizes expected in the flow through the IRT test section and the optical path lengths through the IRT measurement section, a single scattering model can be applied $^{2}$. Thus, the extinction of a light beam passing through the spray will be proportional to the line integral of liquid water content along the path of the ray through the test section.

That is, if $I_{0}$ denotes the intensity of the light source, $I$ denotes the intensity measured on a detector while the spray is active, and $\mu(x)$ is the attenuation coefficient for the spray, then the data collection model is given by Equation 1.

$$
-\ln \left(\frac{I}{I o}\right)=\int_{l i} \mu(x) d s
$$


The data are measured for a collection of lines $l_{i}$, with each line corresponding to a source-detector pair. From the measured data, the line integral of the attenuation coefficient along the optical path from source to detector is computed. The tomographic reconstruction algorithm is used to obtain the attenuation coefficient from its line integrals. The recovered attenuation coefficient $\mu$ is proportional to the liquid water content. Here, $s$ denotes length along the optical path $l_{i}$ from source to detector. Measurements for $I_{0}$ for each source-detector pair are obtained by running a reference acquisition without an active spray.

The IRT tomography system shares a fundamental measurement model with both medical computed tomography (CT) and the PSL optical tomography systems. However, because of the rectangular cross-section of the IRT, the sources and detectors are arranged on a rectangular frame, as opposed to along a circle for both medical CT and the PSL system. This causes the geometry of the acquisition lines to differ radically from that of those systems. Classical tomography algorithms rely on regular line sampling. The rectangular geometry yields an irregular sampling. Due to the relatively low sampling density, there isn't sufficient data to interpolate back to a regular sampling pattern, so traditional tomographic reconstruction algorithms, even as modified for the PSL system, cannot be applied. On the other hand, the IRT reconstruction requirements and data characteristics closely match those of the PSL system. The region of interest is the entire area of the rectangle, though again the central region is of primary importance. Sparse data sampling implies that reconstructions will be of relatively low resolution. The sprays to be imaged are not expected to have sharp transitions, so the resolution should be sufficient.

The approach for the IRT's system was to apply a variant of the linear fit algorithm that was first proposed by Izen, Bencic $^{3}$ and used in the PSL system ${ }^{1}$. The acquired spray measurements are fit to a linear combination of the simulated measurements of image basis elements. These image basis elements can be pixel based or, as in the case of the IRT, Gaussian blob based. Gaussian blobs better represent the a priori smoothness of the spray. This method is easily adapted to handle minor malfunctions in the acquisition system such as a dead source or a dead detector. The algorithm can also be extended to account for individual source and detector characteristics.

\section{B. Prototype Tomography System Description}

The prototype light extinction tomography system demonstrated in the Icing Research Tunnel consisted of sixty (60) laser diodes with sheet generating optics spaced every six degrees around the periphery of a $6 \mathrm{ft}$ by $9 \mathrm{ft}$ frame structure. In addition, one hundred twenty (120) fiber optically coupled detection elements were mounted every three degrees around this same structure. The conceptual layout of the system is shown in Figure 3 and the actual system set up in the lab for development work is shown in Figure 4a. A close up of the laser diodes and associated light detection elements is shown in Figure 4b.

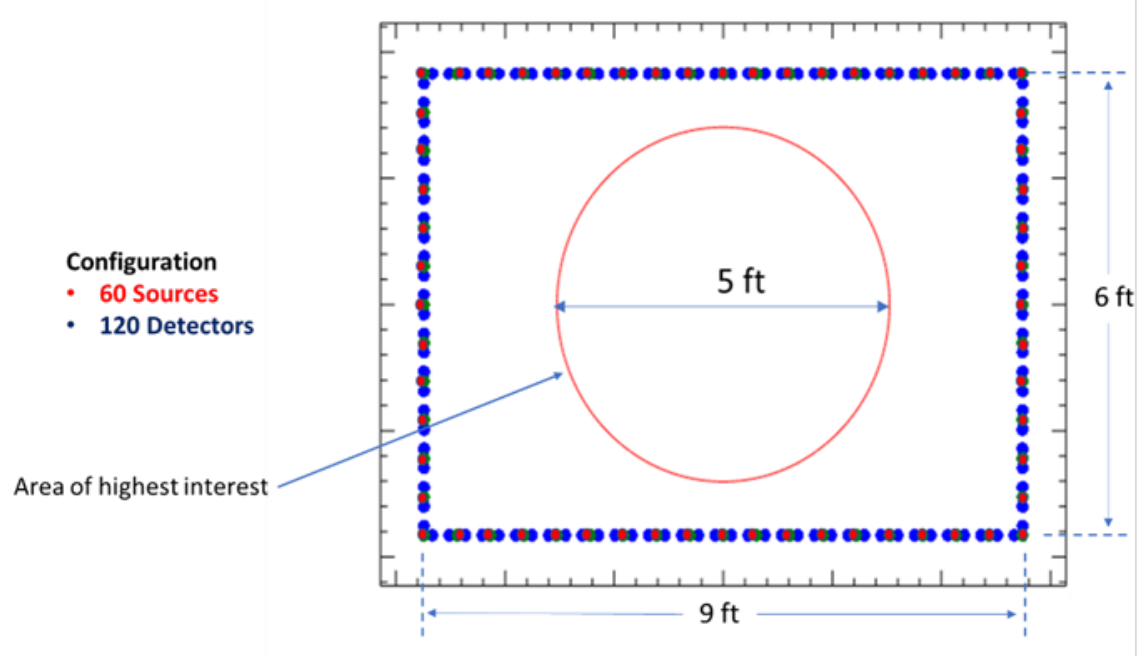

Figure 3. - Conceptual layout of the prototype IRT Light Extinction Tomography System. 


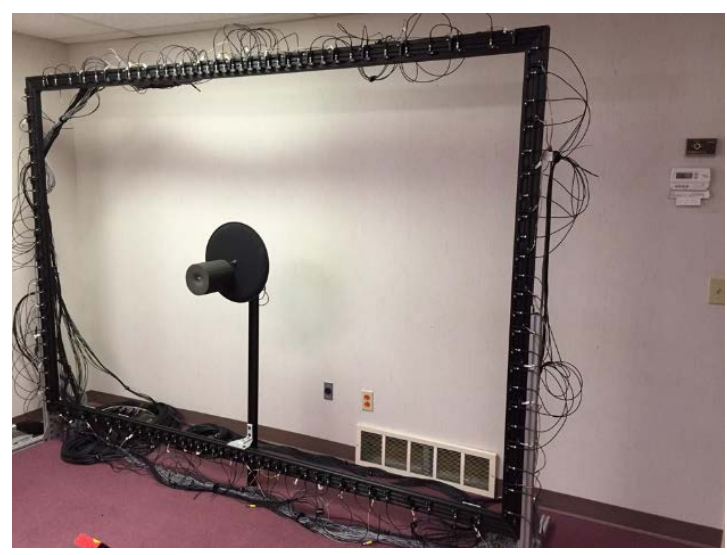

(a)

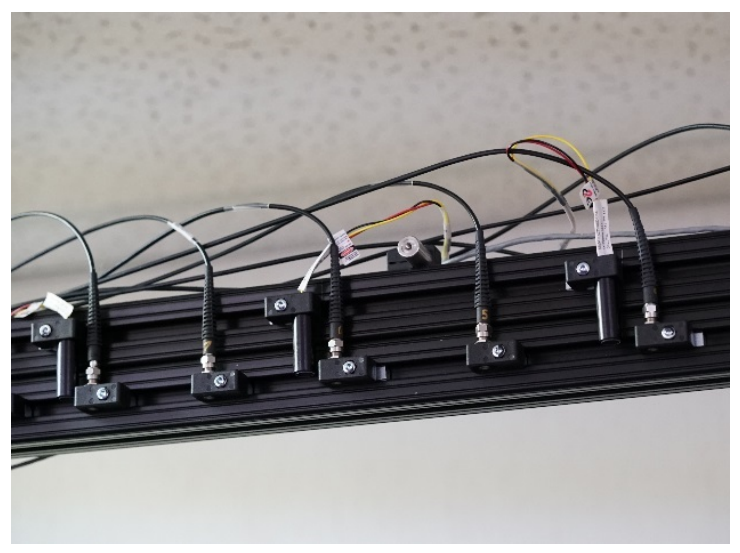

(b)

Figure 4. - Prototype IRT Light Extinction Tomography System. (a) Lab setup (b) Laser diodes and light detectors.

Each detector utilized a flashed opal input diffuser at the fiber entrance which is coupled to the CCD camera for simultaneous sampling of all 120 light detector channels. The diffuser allows coupling of the laser light into the fibers at a very wide input angle of approximately $+/-85$ degrees with respect to the fiber face. The diffusers greatly increase the acceptance angle of the fibers at the cost of allowing only a small amount of the incident light to be coupled into the fiber. The laser diode sources (658nm wavelength) are pulsed sequentially while the detectors acquire line-of-sight extinction data for each laser pulse. A custom timing/triggering circuit was built in-house and used to pulse the laser diodes and control the data acquisition. The optical fibers are directly coupled to the CCD camera through a fiber optic faceplate. The fibers are directly butted up to the CCD to give a simultaneous snap shot of what the detectors are seeing for a given laser pulse. Two different CCD camera configurations were investigated for the purposes of reading the detector data for this prototype system. The first was a line scan camera with 12 bit depth (Fig. 5a). The second was a traditional area scan camera with 16 bit depth (Fig. 5b). Computed tomography algorithms were then used to reconstruct the data acquired from the detectors to provide a two dimensional image of the uniformity of the cloud flow through the test section. It was envisioned that the line scan camera would decrease the read time and result in faster response time. However, during the demonstration testing in the facility it was found that the area scan camera with its 16 bit depth provided the best overall results and will be used for future implementations of the system. More on this will be discussed in the following sections of this paper.

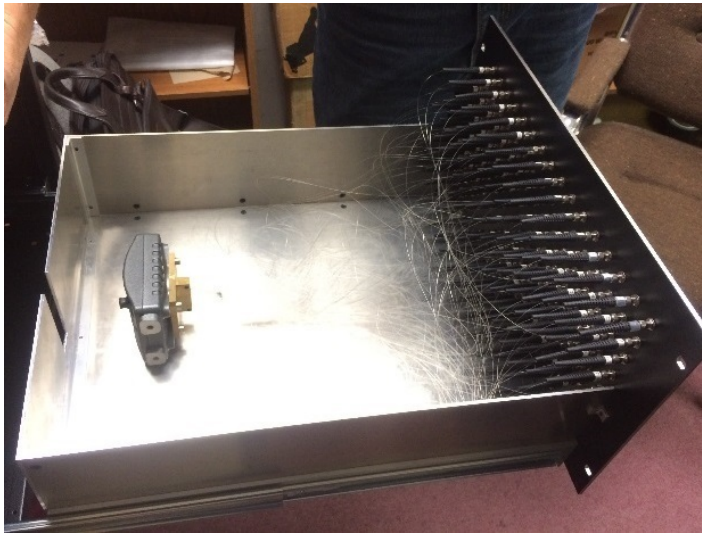

(a)

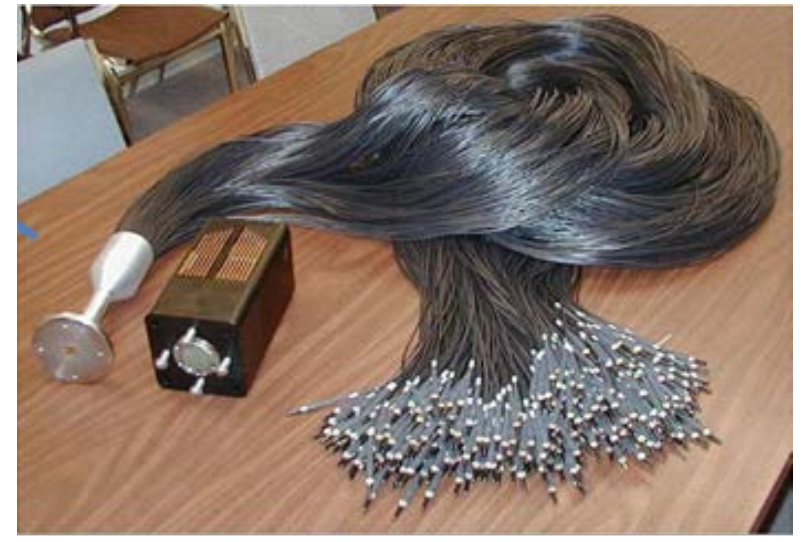

(b)

Figure 5. - Camera Configurations. (a) Line scan camera to fiber interface (b) Area scan camera to fiber interface. 


\section{Facility Installation}

The main intent of this initial demonstration in the IRT facility was to serve as a proof-of-concept test to assess if the tomographic acquisition and reconstruction principles used for this large $6 \mathrm{ft}$ by $9 \mathrm{ft}$ geometry would be able to make the required uniformity and density measurements in the IRT before investing additional funds and design resources required for further refinement and permanent installation of this system into the facility's test section. With this in mind, a decision was made to use the existing prototype system's off-the-shelf rail hardware and mount it downstream of the test section in a location where it would fit as opposed to building a second rig using custom fabricated lower profile hardware that would fit inside the test section. This approach saved both time and money in accomplishing this initial proof-of-concept demonstration in the facility while not significantly compromising the overall facility icing cloud conditions that were to be measured.

The prototype system was installed in the facility diffuser, 91 in. downstream from the end of the test section as this was found to be the location that would accommodate the prototype hardware's outside width and height while still being close to the test section. The conceptual layout of the prototype system is shown in Figure 6 . The frame was bolted to the tunnel walls through the leveling feet that were installed to properly position the structure. Custom fabricated aluminum fairing panels were installed on both the upstream and downstream side of the tomography system's frame to guide the flow over and through the system along with providing protection to the optics and cabling. To prevent ice from building up on the optics and to further minimize flow disturbances, acrylic panels were installed along the periphery of the rig in the measurement plane where the optical access slots were located. Pictures showing the system during various steps of the installation are shown in Figures $7 \mathrm{a}$ and $7 \mathrm{~b}$. The system fully installed and ready for test operations is shown in Figure 7c.

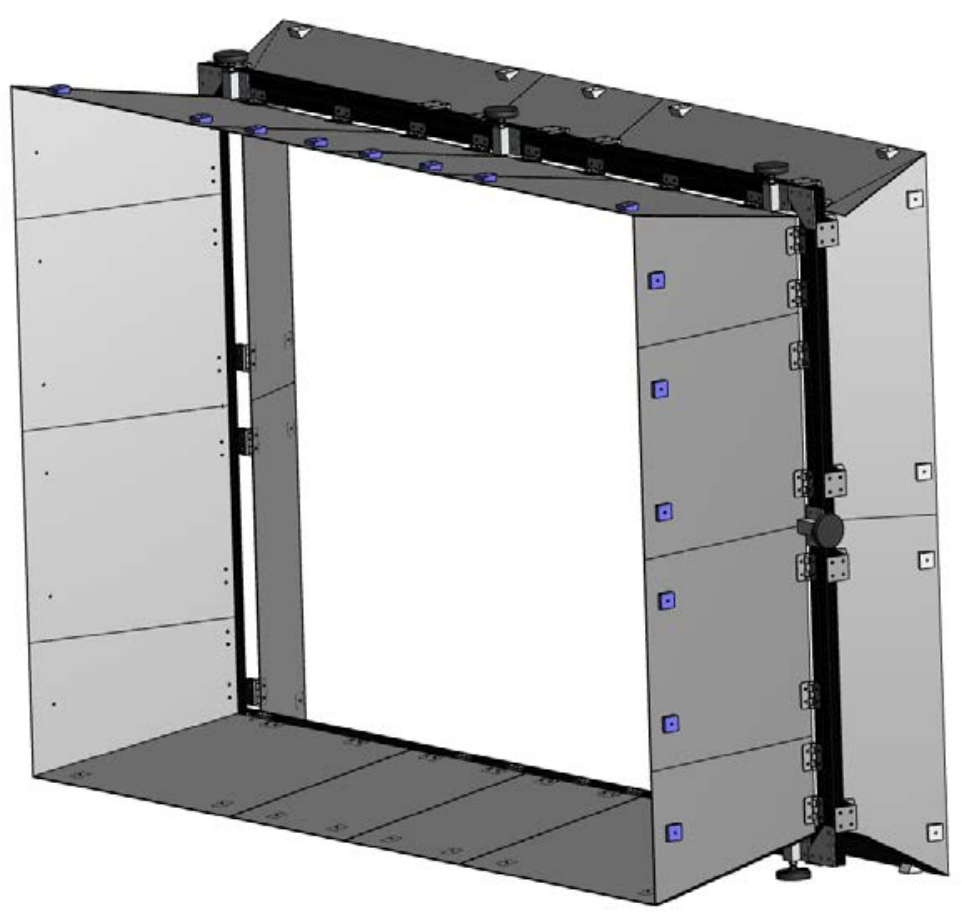

Figure 6. - Conceptual facility installation. 


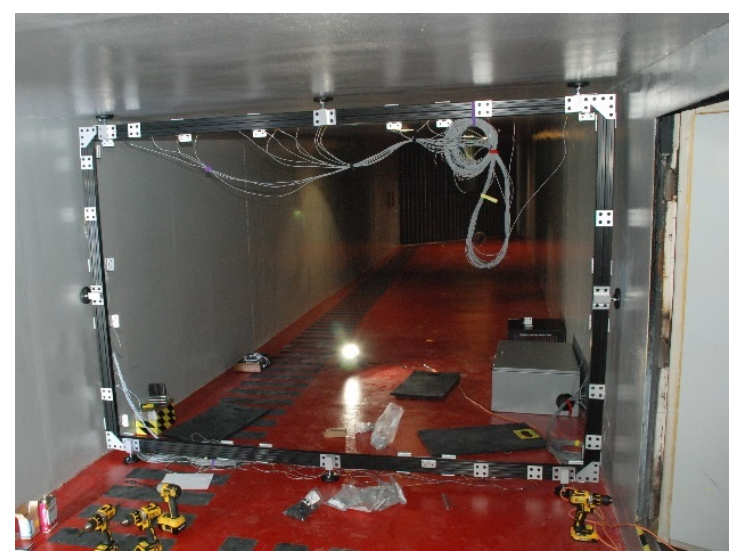

(a)

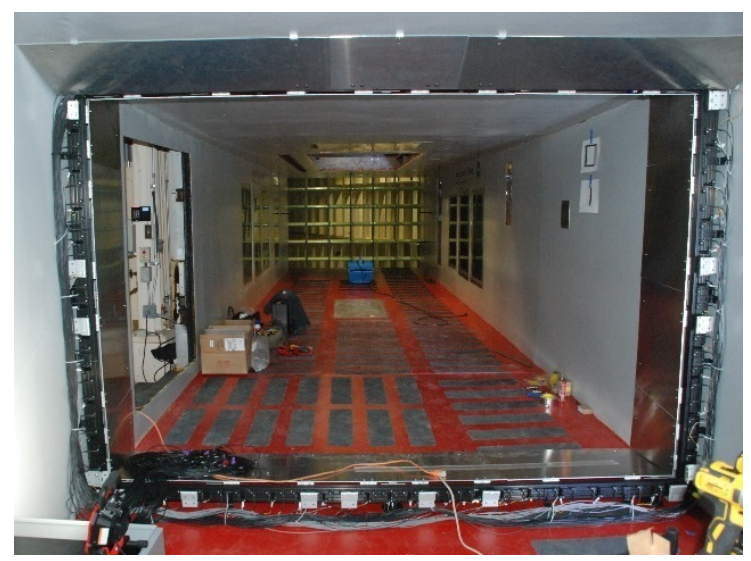

(b)

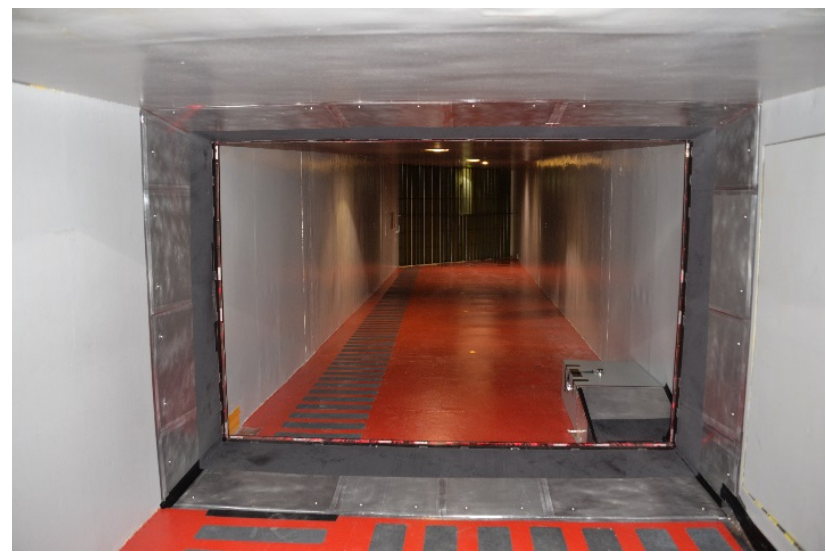

(c)

Figure 7. - Light extinction tomography system installation. (a) Frame being mounted in facility diffuser, looking downstream (b) Forward fairings installed, routing of laser and fiber optic cables on aft side in process, looking upstream (c) Installation complete, system ready for test operations, looking downstream.

In viewing the pictures of Figure 7, one can see that there were many compromises made in the installation for this initial proof-of-concept test. First, the off-the-shelf lab hardware did not have the low flow profile that will be needed in the final permanent version. This not only drove the location where it could be located in the facility, but it also created the need for the aluminum fairings on both the upstream and downstream side of the system to minimizes flow disturbances and guide the flow over the hardware. This created a convergent-divergent area at the system that would not be present in the permanent version. Second, the lab hardware had limited lengths of cables for the lasers and fiber optic light detectors. This caused the controller for the laser and the camera for the light detectors to be located in a metal cabinet that was installed downstream of the system in the diffuser. This is shown on the lower right-hand corner of the system in Figure 7c. This had the potential to create a localized flow disturbance in this corner which would not be normally present in the facility's flow. For the permanent installation these components will be located outside of the test section with sufficient lengths of cabling and penetrations required to route the cabling through the test section walls, minimizing the amount of hardware in the flow path. In the end, even with these compromises, the ultimate goal of assessing the system's performance for this initial proof-of-concept test was achieved. It was felt by the authors' that it was better to validate the system's performance before pursuing a more costly installation that would eliminate all of these issues. These items will be addressed in the final system design for the permanent installation of this system into the IRT. 


\section{Demonstration Test Plan and Execution}

To accomplish the demonstration testing of the prototype light extinction tomography system a test matrix was developed that would operate the IRT facility's spray bars over their operational range and provide conditions that would be appropriate for assessing the system's ability to detect cloud location, visualize cloud uniformity, and detect minute changes in cloud uniformity due to changes in the spray system. The test matrix and conditions that were used for the demonstration testing in the IRT are shown in Appendix A. From analyzing the matrix one can see that testing was accomplished at a facility speed of 150 knots. The droplet size, liquid water content, pattern, nozzles, and nozzle spray pressures used for the icing sprays were varied to provide the following main categories of test conditions: Distortion Checks, Baseline Grid Sprays, Sensitivity Checks, and Tomographic Exercises.

The Distortion Checks were conducted by turning on only a specific column or row of the spray bar system to see if the pattern would show up in the correct location of the tomography system's two-dimensional measurement plane. The purpose of this series of conditions was two-fold. First, they were conducted to make sure that the icing cloud's location was not grossly changing due to being measured in the diffuser as opposed to the test section, where cloud patterns in relation to spray bars are known through previous calibrations. Second, it served as a good overall test of the system's ability to correctly track the expected cloud spray locations as a function of spray bars.

The Baseline Grid Spray conditions were conducted by operating the spray bar at known calibrated baseline conditions using the Standard or Mod1 spray nozzles. These are the conditions for which the IRT regularly manually measures for cloud uniformity, and are very similar to the conditions used to generate icing clouds for customer's tests. The purpose of these tests was to see how cloud uniformity measured by the light extinction tomography compared to uniformity data acquired from previous calibrations. These test conditions were repeated several times to determine how repeatable the system was in making these known cloud uniformity measurements.

The Sensitivity Checks involved operating the spray bars at the known calibrated configuration, but varying the median volumetric diameter (MVD) and liquid water content (LWC) of the cloud to determine if the tomography system could detect the changes in cloud uniformity.

Lastly, the Tomographic Exercises were conducted by operating at the calibrated spray conditions (Standard and Mod1) but involved turning off and on various patterns of nozzles to simulate faults that may happen in the spray bar system changing the spray from its known calibrated conditions. These tests were conducted to determine if the tomography system was sensitive enough to detect these small changes. This is important, because in the eventual system, the primary use will be to monitor cloud uniformity in-situ to provide a means to detect any variations due to anomalies in the spray system.

As indicated by the test matrix in Appendix A, data sets were acquired for 79 icing cloud spray conditions over three days of test operations in the IRT facility. During test operations several anomalies were encountered. These anomalies were successfully addressed and are summarized below.

First, it was originally intended to take the facility up to its full speed condition and acquire data at 220 knots. However, it was found that while operating at this high speed condition, tape that was used to seal up the fairing seams on the tomographic system kept coming off. There was also flexing observed in the upstream aluminum fairing panels. It was decided that, to eliminate these undesirable issues for this demonstration test, data would be taken at 150 knots as this was a known facility condition with calibrated cloud uniformity data. This was thought to be sufficient for this proof-of-concept test. The use of tape and temporary fairings will not be an issue in the permanent facility system. During the first day of testing it was found that the particular line scan camera that was being used did not provide the sensitivity that would be required to properly measure the icing cloud conditions. In addition, the camera was having trouble working in the environmental conditions associated with the test facility. To address this the line scan camera was removed and replaced with the area scan camera. The area scan camera operated flawlessly and was used to acquire data for all subsequent testing on the second and third days. The line scan camera will not be used in the future implementations of the system. Also during test operations on the first day, it was found that condensation was building up on the inside of the Plexiglas that was covering the optics. This was corrected by adding air lines to these cavities to provide a small amount of purge air to remove the condensation. Lastly, it was found during end of test operations for a given day, several of the sheet-generating laser diodes had 
become misaligned, requiring realignment prior to the next day's testing. This was thought to be due to vibrations being introduced on to the tomographic systems frame during testing. This issue will be better addressed in the permanent system's design. Again, it needs to be stressed that all of the anomalies listed above were minor solvable issues and did not take away from the successful demonstration of the prototype tomographic light extinction system in the IRT. All of these issues will be taken into account in the final system design.

\section{Preliminary Results and Analysis}

Since this was a proof-of-concept test to determine if the technique was viable in detecting, reconstructing and mapping the icing cloud in the Icing Research Tunnel, only a qualitative analysis was conducted. The relative uniformity and density of the clouds, as measured by the aforementioned manual methods, were compared to the reconstructed tomographic intensity data mapped onto a two-dimensional image plane that represents the cross section of the measurement plane in the facility. This provided a good visual means of assessing cloud shape and uniformity. It is the eventual goal to use this system to provide quantitative density and liquid water content measurements by using physical probes and relating their measurements to image intensity. This technique has been successfully employed in the Propulsion Systems Laboratory ${ }^{1}$. It will be pursued in future implementations in the IRT facility. A subset of the data acquired for each testing category along with a brief analysis discussion is presented below.

\section{A. Distortion Checks}

Figure 8 shows the cloud maps that were acquired for three of the Distortion Check test conditions. In these three cases only a particular horizontal row of spray bars (lower, middle, and upper) were turned on to see if the expected patterns would show up in the correct location on the light extinction tomography system. As mentioned previously, the intent of this test was to not only verify that the system was correctly tracking the icing flow, but also to verify that the icing cloud's location was not grossly changing while being measured in the diffuser as opposed to the test section where all of the previous data had been acquired. It can be observed in Figure 8 that the location of the spray bar that is being operated (lower, middle, and upper) shows up in the expected location on the two-dimensional tomographic images. Similar patterns were also correctly observed when only specific vertical columns of spray bars were activated, hence further verifying that the icing spray was operating as expected and the tomography system was correctly measuring the cloud's location.

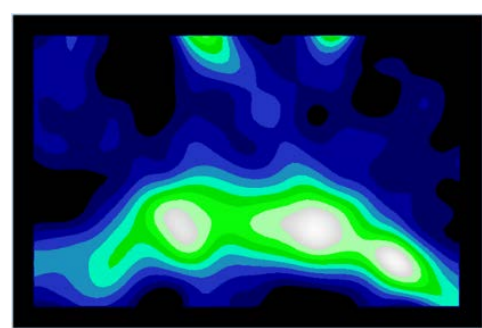

(a)

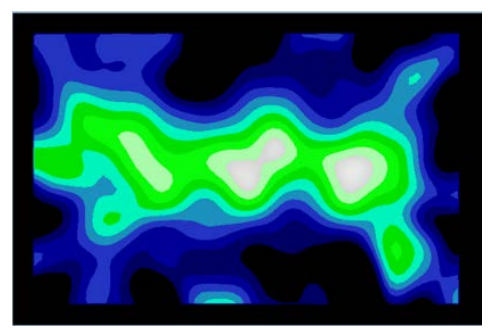

(b)

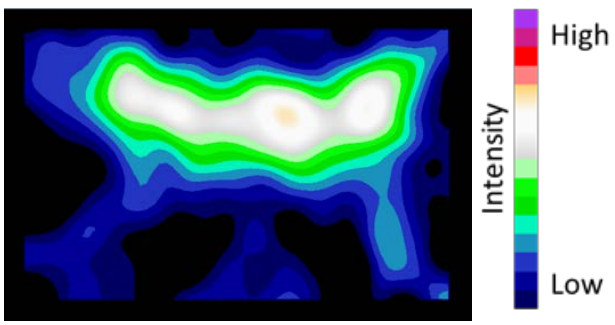

(c)

Figure 8. - Tomography data acquired during Distortion Checks. (a) Lower spray bar only operation (b) Middle spray bar only operation (c) Upper spray bar only operation.

\section{B. Baseline Grid Sprays}

The baseline grid sprays (Standard and Mod1) were conducted to determine how well the baseline grid spray uniformity, as measured by the tomography system, matched the uniformity data acquired from previous tunnel calibrations. In addition, several measurements of the same spray conditions were acquired at different times to assess variation in the cloud uniformity as measured by the system. The mean tomographic reconstruction for the standard baseline spray conditions is shown in Figure 9a. This mean was developed by averaging the results of the seven Standard baseline grid spray test runs that were conducted during the demonstration test (runs 29, 33, 43, 45, 55, 68, 
and 75). The intensity values of this mean reconstruction were normalized to the intensity values located at the centerline of the test section. This was done in order to provide a better comparison to the LWC calibration plot (Fig. 9c) that was derived from a facility calibration conducted in January 2018, one month previous to this demonstration, using the manual icing measurement grid. This uniformity plot employs a similar type of normalization. The standard deviation for the seven standard baseline sprays measurements is shown in Figure 9b. The red dashed box in the plots of Figures 9a and 9b corresponds to the comparative cross sectional area of the manually-measured grid and denotes the primary area of interest for analysis.

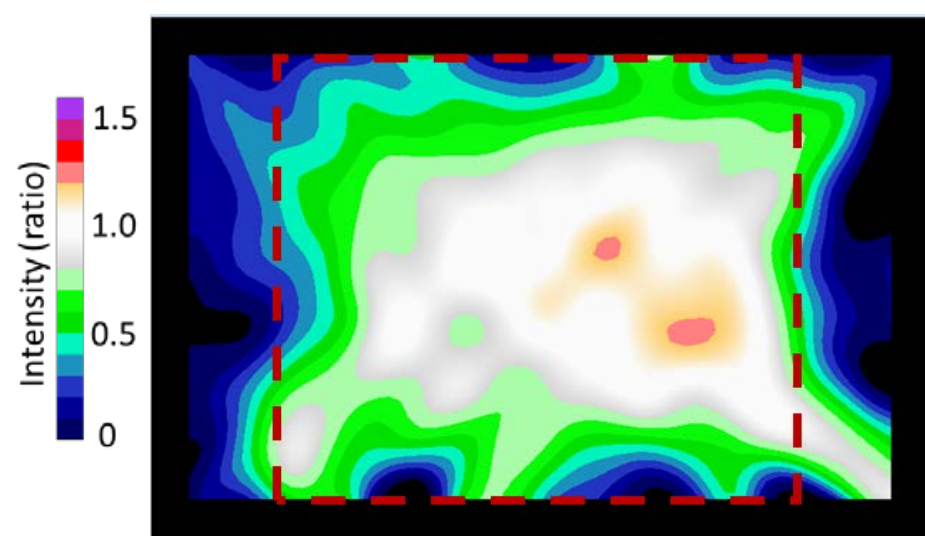

(a)

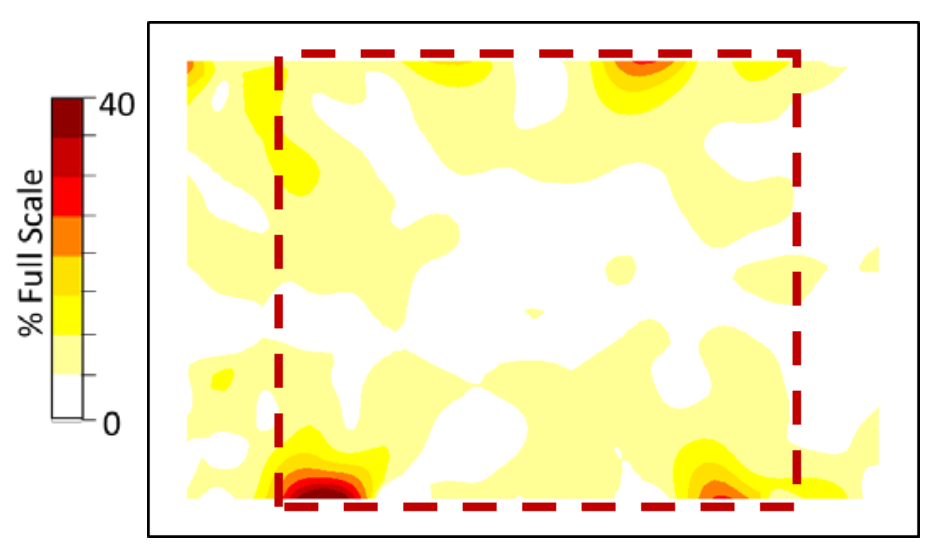

(b)

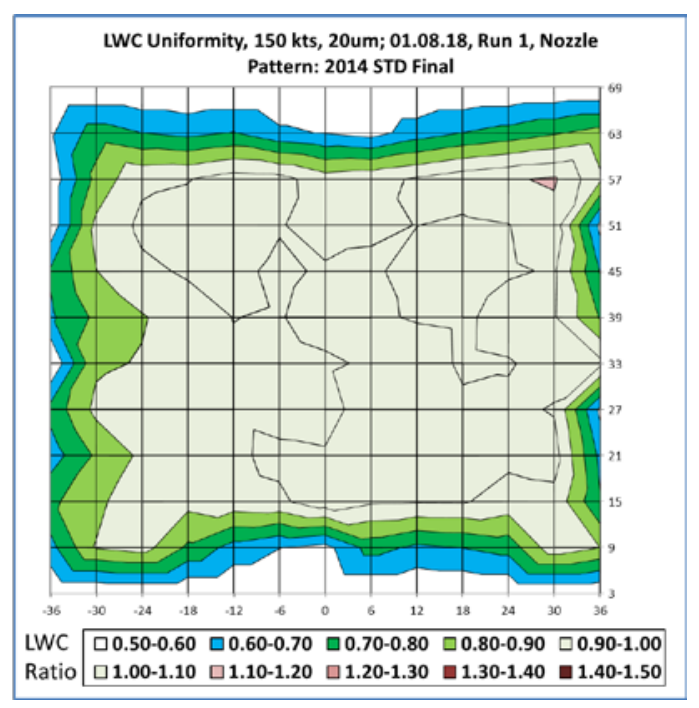

(c)

Figure 9. Standard baseline grid spray results. (a) Mean tomographic reconstruction (b) Standard deviation of tomographic reconstructions (c) Calibration grid map from Jan 2018.

From comparing the mean average of the Standard baseline grid spray as measured by tomography system (Fig. 9a) to the calibration map that was obtained from using the grid (Fig. 9c) it is evident that the results closely follow the same trends and have a similar shape. Each plot shows icing cloud uniformity with ratio values of 0.90 to 1.10 within the center core section of the tunnel flow. The tomographic system's reconstruction does trend towards lower ratio values ( 0.70 to 0.80$)$ along the edges of the core flow regime when compared to the calibration map's ratios. In addition, the tomographic results show two high spots (ratios of $\sim 1.2$ ) in the core flow region that do not appear in the calibration map. These differences can be attributed to several reasons. First, the calibration map was generated by using the grid that is located in the test section as opposed to the diffuser section where the tomographic system was installed. The conditions in the diffuser may be slightly different than the condition previously measured in the test section. Uniformity data has not been previously acquired in the diffuser location. In addition, as mentioned in Section III, effects associated with the prototype tomographic system's temporary installation in the diffuser such as convergent-divergent section caused by use of the aluminum fairing panels and the installation of the equipment box just downstream of the measurement plane may have contributed to the differences that were observed.

American Institute of Aeronautics and Astronautics 
In regards to measurement variability between the data sets acquired during the seven baseline grid spray test runs, the standard deviation plot (Fig. 9b) shows 0 to $10 \%$ reading-to-reading variation in the center core section of the tunnel flow. This is within reason and was expected. The high variations at the outer edges are not of concern as this is a common observance with tomographic reconstructions. Artifacts like this occur at the edge of the measurement plane when a laser or light detector is out of alignment for one or more of the test runs. While this may create an undesired anomaly on the edge of the measurement plane, the system is robust enough to yield good measurements in the desired core center section where the tunnel flow is of most concern.

Similar plots for the Mod1 baseline spray conditions are shown in Figure 10. The mean tomographic reconstruction is shown in Figure 10a. This mean was developed by averaging the results of the seven Mod1 baseline grid spray test runs (30, 32, 42, 44, 54, 67, and 74). The standard deviation for these data sets is shown in Figure 10b. As in the previous case, the LWC uniformity map that was generated during the tunnel calibration in January 2018 is included for comparison in Figure 10c. The shape and uniformity of the data acquired with the tomography system shows similarities to that of the calibration map. However in this case, the calibration map actually shows more high and low spots than what appear on the tomographic reconstruction. As in the Standard baseline spray case, this may be due to the difference in the locations where these measurements were made (test section vs diffuser). Also, as there is about half the liquid water content in this spray, other effects associated with the temporary installation may be limiting the system sensitivity needed to fully visualize this lighter spray condition. Increasing system sensitivity will be further investigated in the final system design. The reading-to-reading variability in the center core flow region, as depicted in the standard deviation plot (Fig. 10b), shows a range of 0 to $10 \%$ full scale, which was similar to the Standard spray case.

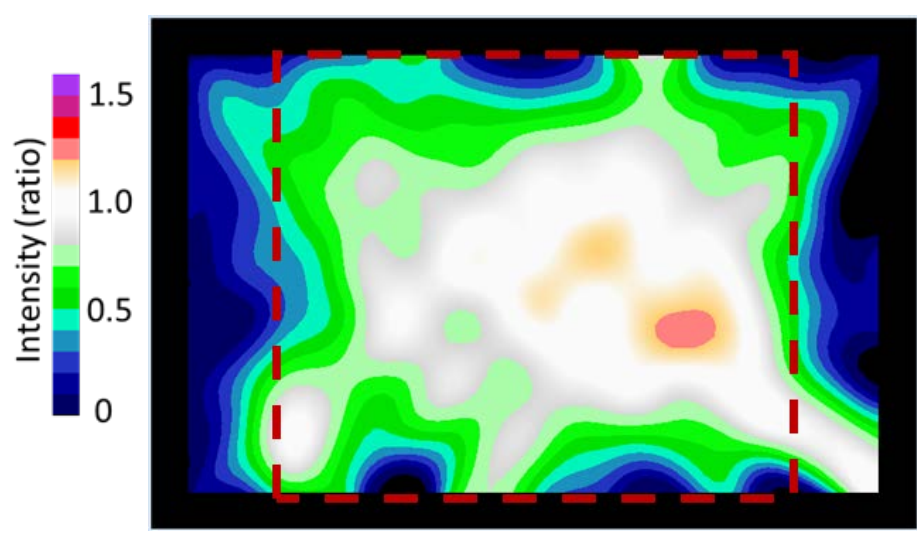

(a)

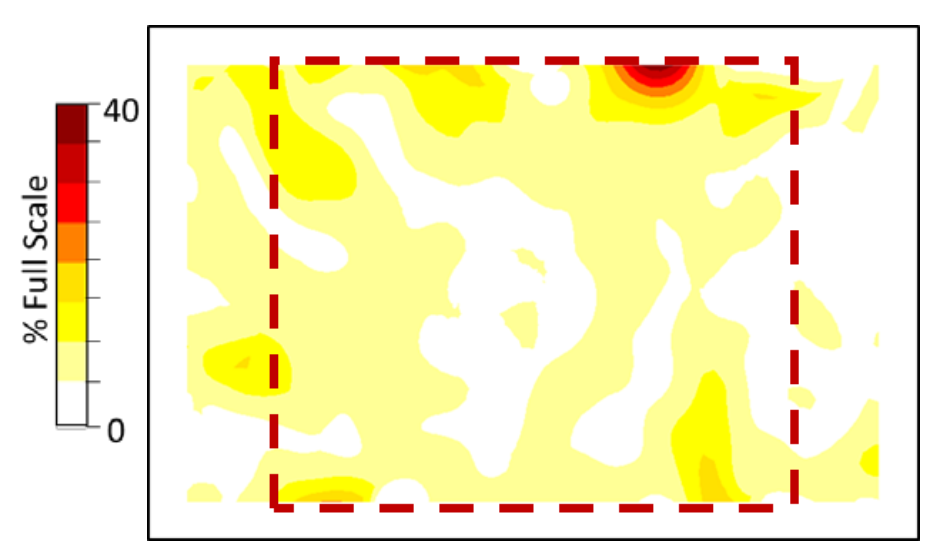

(b)

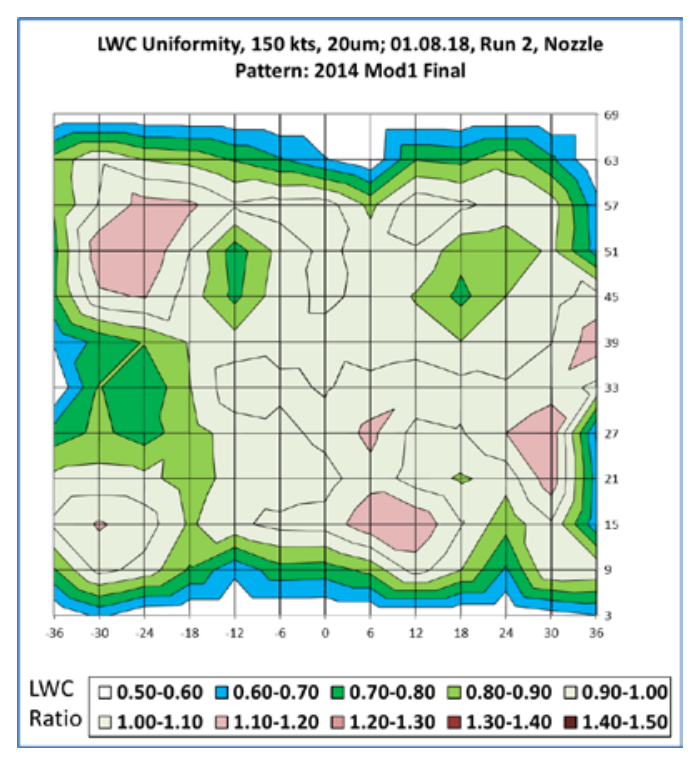

(c)

Figure 10. Mod1 baseline grid spray results. (a) Mean tomographic reconstruction (b) Standard deviation of tomographic reconstructions (c) Calibration grid map from Jan 2018.

American Institute of Aeronautics and Astronautics 
One thing that would have helped better resolve these observed differences would have been to install the calibration grid just downstream of the light extinction tomography system during the demonstration test. This would have yielded a true one-to-one comparison. This was not possible during the time of the test because there were not provisions to easily install the grid in the diffuser within our allocated resources and timeframe. This installation will be considered in future demonstration tests planned for the system in the IRT. However, regardless of these slight differences, the Standard and Mod1 baseline grid sprays as measured by the tomography system reasonably matched the overall shape of the uniformity plots and the ratio measurements were in-line with those acquired with the manually measured grid. Thus, the tomographic technique has high potential to make the desired baseline cloud uniformity measurements in the IRT.

\section{Sensitivity Checks}

As described previously, the Sensitivity Checks involved operating the spray bars at the known calibrated configuration, but varying the MVD and LWC of the spray to determine if the tomography system could detect the change in cloud uniformity. Figure 11 shows the results acquired during several test runs of the Mod 1 spray where the MVD was varied over a range of known configurations.

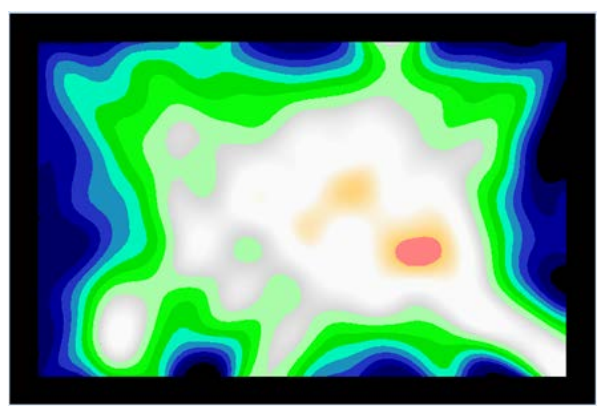

(a) Mod1 Baseline, MVD=21.5 $\mu \mathrm{m}, \mathrm{LWC}=0.70 \mathrm{~g} / \mathrm{m}^{3}$

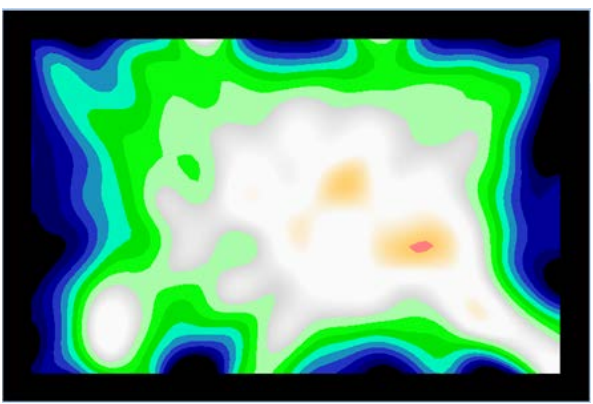

(b) Mod1, MVD=15.3 $\mu \mathrm{m}, \mathrm{LWC}=0.43 \mathrm{~g} / \mathrm{m}^{3}$

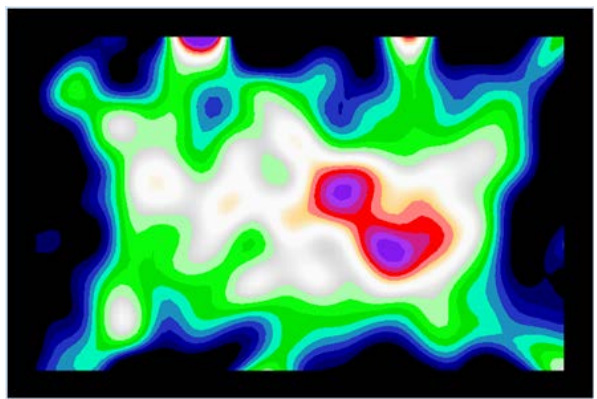

(d) Mod1, MVD=266 $\mu \mathrm{m}$, LWC $=0.92 \mathrm{~g} / \mathrm{m}^{3}$

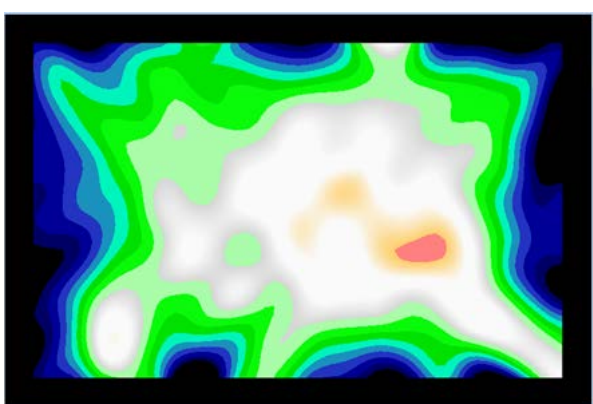

(c) Mod1, MVD=40.9 $\mu \mathrm{m}$, LWC=1.19 g/m $\mathrm{m}^{3}$

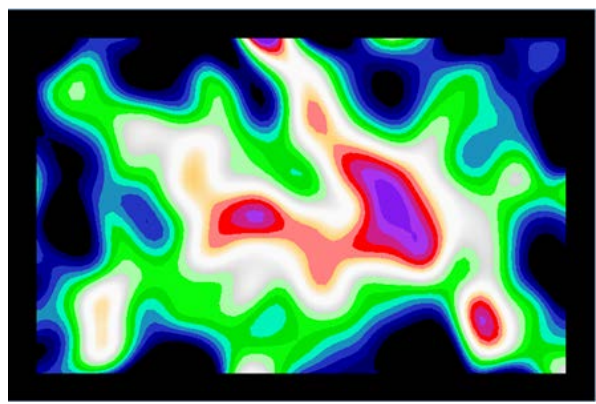

(d) Mod1, MVD=460 $\mu \mathrm{m}, \mathrm{LWC}=1.26 \mathrm{~g} / \mathrm{m}^{3}$

Figure 11. Mod1 spray variation results. (a) Mean of Mod1 baseline sprays (b) Test run \#48 (c) Test run \#49 (d) Test Run \#52 (e) Test run \#53 
In analyzing Figure 11, minimal changes from the baseline Mod1 spray results with MVD=21.5 $\mu \mathrm{m}$ (Fig. 11a) are observed when the drop MVD is varied from 15 um (Fig. 11b) to $41 \mu \mathrm{m}$ (Fig. 11c). The cloud uniformity and shape as measured by the tomography system for these typical drop sizes are nearly identical. This is consistent with results observed in the previous manual grid measurements obtained in 2014 ${ }^{4}$. However, noticeable variations are observed when the "large drop" size sprays were used (Figs. 11d \& 11e). These test cases involved using sprays that had drop MVDs of $266 \mu \mathrm{m}$ and $460 \mu \mathrm{m}$ respectively. These conditions are on the upper end of the facility's maximum drop diameter capabilities. In these two cases there is a noticeable shrinking of the cloud and higher concentrations in the central portion of the measurement plane. It is surmised that this cloud shrinkage is likely due to both the presence of larger drops which tend more towards test section center and the decreased flow mixing caused by lower nozzle air pressures. This was also consistent with the calibration in 2014. It should be noted that the results shown in Figure 11 are normalized to better assess uniformity. In looking at the raw images the overall intensity values do vary due to changes in overall LWC. This will be critical when the system is used to provide this measurement in addition to assessing uniformity. This will be further investigated in future test entries.

\section{Tomographic Exercises}

The Tomographic Exercises were conducted by operating at the calibrated grid spray conditions with a predetermined pattern of nozzles turned off (or on) to simulate faults that may occur in the system. The goal of these tests was to determine if the tomography system had the sensitivity to detect these anomalies. The tomographic exercise presented in this paper is from test runs 38, 39 and 40. This exercise involved the use of a high number density (ND) Standard spray with a MVD of $15.4 \mu \mathrm{m}$ and a LWC of $1.52 \mathrm{~g} / \mathrm{m}^{3}$ as the baseline reference condition (Fig. 12a). After acquiring the baseline measurement, three of the Standard spray nozzles were turned off to assess if the system could detect the difference that this would create in the spray pattern. The nozzles that were turned off were on the upper left hand side, the center, and the lower right hand side of the spray bar system, forward looking aft. This directly corresponds to the location they would appear in the reconstructed tomographic images in Figure 12. The results acquired for this off-nominal spray condition are shown in Figure 12b. The last part of this exercise involved keeping the three Standard spray nozzles off, and additionally turning on three low flow rate Mod1 spray nozzles that were near the Standard nozzles to further give a disturbance for the purposes of detection. The results of these off-nominal spray conditions are shown in Figure 12c.

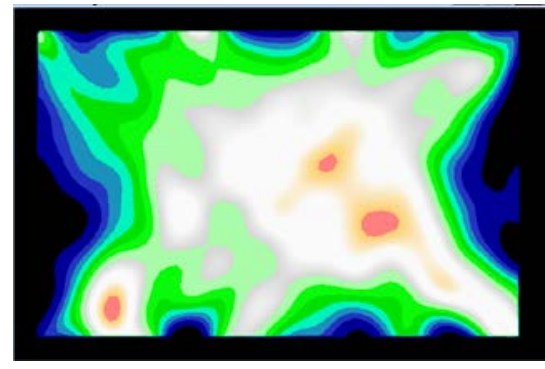

(a)

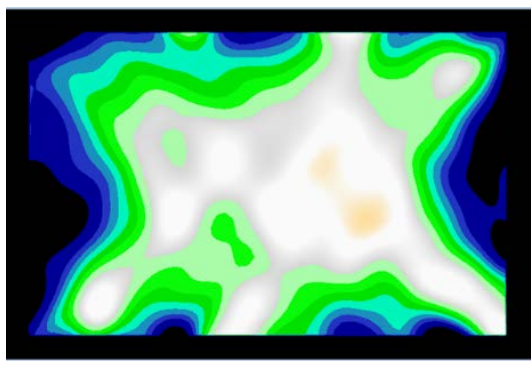

(b)

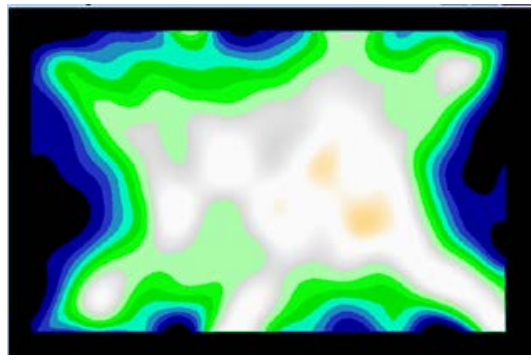

(c)

Figure 12. Tomographic Exercise Results. (a) Run \#38 - High ND Standard-nozzle spray (b) Run \#39 Three Standard nozzles off (c) Run \#40 - Three Standard nozzles off, three Mod1 nozzles on

For analysis, image subtraction was used between the successive test conditions to determine if the system could measure the differences due to the sequencing of the spray nozzles. The difference between the baseline condition (run 38) and the same condition with the three Standard spray nozzles turned off (run 39) is shown in Figure 13a. In analyzing this plot, one could argue that the diagonal pattern that starts on the lower right and ends on the upper left portion of the diagram somewhat matches the location of where the valves have been turned off. However, the results are inconclusive, as the differences observed across the entire core section of the cloud fall within $+/-10 \%$. This is within the measurement repeatability tolerance shown in the previously discussed Baseline Grid Spray analyses. Similarly, the difference between the test condition with the three Standard nozzles off (run 39) and the one with the three Mod1 nozzles turned on (run 40) is shown in Figure 13b. Again, the differences between these readings is +/$10 \%$ across the cloud flow and no real pattern that relates to the valve location is observable. Hence, at least for this 
initial demonstration the system appears not to have the sensitivity needed to detect differences on this small scale. This may be due to the fact that the tomography system is located downstream in the diffuser, allowing additional mixing of the flow to occur, thereby minimizing the "holes" that were expected in the cloud due to the operation of the nozzles. The lack of sensitivity may also be due to the installation effects previously mentioned for this temporary proof-of-concept demonstration. Either way, this issue will be further investigated with the goal of improving system sensitivity for future installation in the facility. For future demonstrations, it is recommended that larger deviations be employed so a better understanding of the system's anomaly detection limits can be realized.
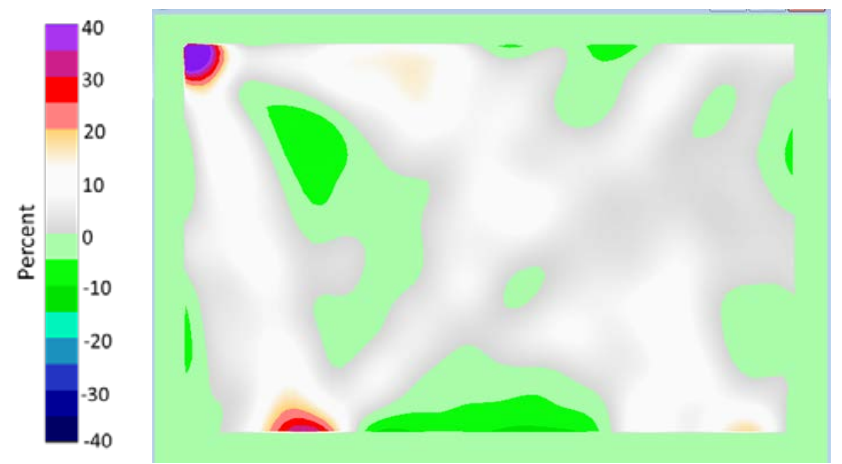

(a)

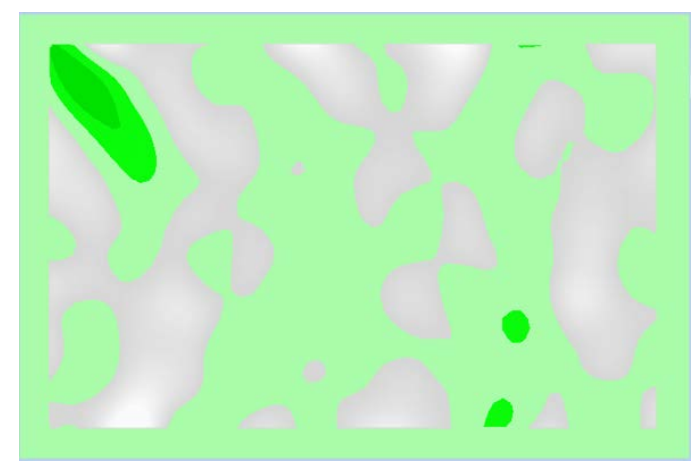

(b)

Figure 13. Tomographic exercise analysis. (a) Run \#38 minus Run \#39 (b) Run \#39 minus Run \#40

\section{Conclusion}

The prototype light extinction tomography system was successfully installed and demonstrated in the NASA Glenn Research Center's Icing Research Tunnel. It was shown that the system could be used to track the icing cloud location in the facility and provide a means of assessing the cloud's shape and uniformity. The results were comparable to previous results acquired from using the manual grid. This was encouraging in that it shows that this technique, which has never been attempted on a square geometry of this large scale, is viable and has potential for future implementation into the Icing Research Tunnel. It was also found that the prototype was limited in being able to detect failures on the single spray nozzle level that were simulated during the demonstration. However, this is thought to be solvable, and may be more due to issues associated with the temporary installation as opposed to limitations of the technique itself. In the end this was a very successful proof-of-concept of the prototype light extinction tomography system. The results acquired in this proof of concept demonstration test will serve as the basis for further improvement and long term installation of a light extinction tomography system in the Icing Research Tunnel.

\section{Acknowledgements}

The authors would like to thank Trevor John for the machining and build-up of the tomography system hardware, the IRT facility engineers and technicians for their dedication and hard work in supporting this test, and IRT Facility Manager Jack Oldenburg in accommodating this system demonstration in the ever busy IRT test schedule. Lastly, the authors would like to acknowledge the NASA Aerosciences Evaluation and Test Capability (AETC) Portfolio's Test Technology Subproject for supporting this effort. 


\section{References}

${ }^{1}$ Bencic, T., Fagan, A., Van Zante, J., Kirkegaard, J., Rohler, D., Izen, S., “Advanced Optical Diagnostics for Ice Crystal Cloud Measurements in the NASA Glenn Propulsion Systems Laboratory,” 5th AIAA Atmospheric and Space Environments Conference, San Diego, CA. June 24-27, 2013

${ }^{2}$ Lock, J., "Mapping the Position-Dependence of the Liquid Water Content in the NASA Glenn Icing Research Tunnel Using Light Scattering Tomography,” NASA Grant NAG3-2232

${ }^{3}$ Izen, S. H. and Bencic, T. J., "Application of the Radon Transform to Calibration of the NASA-Glenn Icing Research Wind Tunnel,” Contemporary Mathematics, Vol. 278, 2001, pp. 147-166.

${ }^{4}$ Steen, L., Ide, R., Van Zante, J., Acosta, W., "NASA Glenn Icing Research Tunnel: 2014 and 2015 Cloud Calibration Procedures and Results,” NASA TM-218758, 2015 
Appendix A. - Test Matrix for IRT Tomography System Demonstration

\begin{tabular}{|c|c|c|c|c|c|c|c|}
\hline & \multicolumn{3}{|c|}{ Facility Setup } & \multicolumn{2}{|c|}{ Cloud Conditions } & \multirow[b]{2}{*}{ Nozzle Set } & \multirow[b]{2}{*}{ Objective } \\
\hline Data Pt & $\begin{array}{c}\text { TTTSC } \\
\pm 1 C\end{array}$ & $\begin{array}{c}\text { TSTSC } \\
{[C]}\end{array}$ & $\begin{array}{l}\text { UTSK } \\
\text { [kts] }\end{array}$ & $\begin{array}{l}\text { MVD } \\
2015 \\
\text { [um] }\end{array}$ & $\begin{array}{c}\text { LWC } \\
2015 \\
{[\mathrm{~g} / \mathrm{m} 3]}\end{array}$ & & \\
\hline \multicolumn{8}{|c|}{ Day 1 - 09Feb18 - Line Scan Camera } \\
\hline \multicolumn{8}{|c|}{ Distortion Checks } \\
\hline 2 & +5 & 2.0 & 150 & 18.0 & 1.05 & Std & Bar 3 only - Distortion Check \\
\hline 3 & +5 & 2.0 & 150 & 19.7 & 2.53 & Std & $\begin{array}{l}\text { Bar } 3 \text { only - Distortion Check; inc } \\
\text { LWC }\end{array}$ \\
\hline 4 & +5 & 2.0 & 150 & 21.4 & 1.39 & Std & $\begin{array}{l}\text { Baseline Std CORE } \\
\text { (Bars } 1 \text { \& } 10 \text { OFF) }\end{array}$ \\
\hline \multicolumn{8}{|c|}{ Baseline Grid Sprays. } \\
\hline 5 & -5 & -8.0 & 150 & 21.4 & 1.39 & Std & Baseline Std (Bars 1 \& 10 OFF) \\
\hline 6 & -5 & -8.0 & 150 & 21.5 & 0.70 & Mod1 & Baseline Mod1 \\
\hline 7 & -5 & -8.0 & 150 & 19.7 & 2.53 & Std & Col 18 only - Distortion Check \\
\hline 8 & -5 & -8.0 & 150 & 19.7 & 2.53 & Std & Bar 8 only - Distortion Check \\
\hline 10 & -5 & -8.0 & 150 & 19.7 & 2.53 & Std & Baseline Std (Bars 1 \& 10 OFF) \\
\hline \multicolumn{8}{|c|}{ Day 2 - 12Feb18 - Area Scan Camera } \\
\hline \multicolumn{8}{|c|}{ Sensitivity Checks } \\
\hline 11 & -5 & -8.0 & 150 & 21.5 & 0.70 & Mod1 & 3 Mod1 Noz, light \\
\hline \multicolumn{8}{|c|}{ Distortion Checks } \\
\hline 12 & -5 & -8.0 & 150 & 19.7 & 2.53 & Std & Bar 3 only (lower, hi LWC) \\
\hline 13 & -5 & -8.0 & 150 & 18.0 & 1.05 & Std & Bar 3 only (lower) \\
\hline 14 & -5 & -8.0 & 150 & 18.0 & 1.05 & Std & Col 18 only (S side) \\
\hline 15 & -5 & -8.0 & 150 & 18.0 & 1.05 & Std & Col 42 only ( $\mathrm{N}$ side) \\
\hline 16 & -5 & -8.0 & 150 & 18.0 & 1.05 & Std & Bar 8 only (upper) \\
\hline 17 & -5 & -8.0 & 150 & 18.0 & 1.05 & Std & Bar 6 only (center) \\
\hline 18 & -5 & -8.0 & 150 & 18.0 & 1.05 & Std & Col 26 only (center) \\
\hline \multicolumn{8}{|c|}{ Baseline Grid Sprays } \\
\hline 19 & -5 & -8.0 & 150 & 21.5 & 0.70 & Mod1 & Baseline Mod1 \\
\hline \multicolumn{8}{|c|}{ Repeat Baseline Grid Sprays } \\
\hline 24 & -5 & -8.0 & 150 & 21.5 & 0.70 & Mod1 & Baseline Mod1 \\
\hline 25 & -5 & -8.0 & 150 & 21.4 & 1.39 & Std & Baseline Std \\
\hline \multicolumn{8}{|c|}{ Tomo Exercise } \\
\hline \multicolumn{8}{|c|}{ Simulate failures around three nozzle clusters: (Std, Mod1): $(3: 16,3: 17),(6: 26,6: 27)$ \& $(10: 42,10: 43)$} \\
\hline 20 & -5 & -8.0 & 150 & 21.5 & 0.70 & Mod1 & Add Air Onlys, 4:42 \& 2:27 \\
\hline 21 & -5 & -8.0 & 150 & 15.3 & 0.20 & Mod1 & Lo Number Density (ND) \\
\hline 22 & -5 & -8.0 & 150 & 15.3 & 0.20 & Mod1 & Lo ND, 3 Mod1s OFF \\
\hline 23 & -5 & -8.0 & 150 & 15.3 & 0.20 & Mod1, Std & $\begin{array}{l}\text { Lo ND, } 3 \text { Mod1s OFF, } 3 \text { Stds ON } \\
\text { (Std MVD = 19.4, LWC }=0.86)\end{array}$ \\
\hline \multicolumn{8}{|c|}{ Day 3 - 13Feb18 - Area Scan Camera } \\
\hline \multicolumn{8}{|c|}{ Sensitivity Check } \\
\hline 26 & -10 & -13.0 & 150 & 21.5 & 0.70 & Mod1 & 3 Mod1 Noz, light \\
\hline 27 & -10 & -13.0 & 150 & 21.5 & 0.70 & Mod1 & 3 Mod1 Noz, light \\
\hline 28 & -10 & -13.0 & 150 & 21.5 & 0.70 & Mod1 & 3 Mod1 Noz, light \\
\hline \multicolumn{8}{|c|}{ Baseline Grid Sprays } \\
\hline 29 & -10 & -13.0 & 150 & 21.4 & 1.39 & Std & Baseline Std \\
\hline 30 & -10 & -13.0 & 150 & 21.5 & 0.70 & Mod1 & Baseline Mod1 \\
\hline 31 & -10 & -13.0 & 150 & 126 & 0.62 & Mod1 & SLD Baseline \\
\hline \multicolumn{8}{|c|}{ Repeat Baseline Grid Sprays } \\
\hline 32 & -10 & -13.0 & 150 & 21.5 & 0.70 & Mod1 & Baseline Mod1 \\
\hline 33 & -10 & -13.0 & 150 & 21.4 & 1.39 & Std & Baseline Std \\
\hline Tomo E & Exercise & & & & & & \\
\hline Simulate & failures & around & hree $n$ & ozzle clus & $:(S t d, M$ & d1): $(3: 16$, & $3: 17),(6: 26,6: 27) \&(10: 42,10: 43)$ \\
\hline 34 & -10 & -13.0 & 150 & 21.5 & 0.70 & Mod1 & Add Air Onlys, 4:42 \& 2:27 \\
\hline 35 & -10 & -13.0 & 150 & 15.3 & 0.20 & Mod1 & Lo Number Density (ND) \\
\hline 36 & -10 & -13.0 & 150 & 15.3 & 0.20 & Mod1 & Lo ND, 3 Mod1s OFF \\
\hline 37 & -10 & -13.0 & 150 & 15.3 & 0.20 & Mod1, Std & $\begin{array}{l}\text { Lo ND, } 3 \text { Mod1s OFF, } 3 \text { Stds ON } \\
\text { (Std MVD }=19.4, \text { LWC }=0.86)\end{array}$ \\
\hline 41 & -10 & -13.0 & 150 & 15.3 & 0.20 & Mod1, Std & $\begin{array}{l}\text { Lo ND, } 3 \text { Mod1s OFF, } 3 \text { Stds ON } \\
(\text { Std MVD }=19.4, L W C=0.86)\end{array}$ \\
\hline 38 & -10 & -13.0 & 150 & 15.4 & 1.52 & Std & Hi ND \\
\hline
\end{tabular}

American Institute of Aeronautics and Astronautics 
Appendix A (continued). - Test Matrix for IRT Tomography System Demonstration

\begin{tabular}{|c|c|c|c|c|c|c|c|}
\hline & \multicolumn{3}{|c|}{ Facility Setup } & \multicolumn{2}{|c|}{ Cloud Conditions } & \multirow[b]{2}{*}{ Nozzle Set } & \multirow[b]{2}{*}{ Objective } \\
\hline \begin{tabular}{|l|} 
Test Run \\
(Data Pt)
\end{tabular} & $\begin{array}{c}\text { TTTSC } \\
\pm 1 \mathrm{C}\end{array}$ & $\begin{array}{c}\text { TSTSC } \\
{[C]}\end{array}$ & $\begin{array}{l}\text { UTSK } \\
\text { [kts] }\end{array}$ & $\begin{array}{l}\text { MVD } \\
2015 \\
\text { [um] }\end{array}$ & $\begin{array}{c}\text { LWC } \\
2015 \\
{[\mathrm{~g} / \mathrm{m} 3]}\end{array}$ & & \\
\hline 39 & -10 & -13.0 & 150 & 15.4 & 1.52 & Std & Hi ND, 3 Stds OFF \\
\hline 40 & -10 & -13.0 & 150 & 15.4 & 1.52 & Std, Mod1 & $\begin{array}{l}\text { Hi ND, } 3 \text { Stds OFF, } 3 \text { Mod1s ON } \\
\text { (Mod1 MVD = 14, LWC }=0.64 \text { ) }\end{array}$ \\
\hline \multicolumn{8}{|c|}{ Repeat Baseline Grid Sprays } \\
\hline 42 & -10 & -13.0 & 150 & 21.5 & 0.70 & Mod1 & Baseline Mod1 \\
\hline 43 & -10 & -13.0 & 150 & 21.4 & 1.39 & Std & Baseline Std \\
\hline 44 & -10 & -13.0 & 150 & 21.5 & 0.70 & Mod1 & Baseline Mod1 \\
\hline 45 & -10 & -13.0 & 150 & 21.4 & 1.39 & Std & Baseline Std \\
\hline \multicolumn{8}{|c|}{ Bonus Baseline (Additional Sensitivity Checks) } \\
\hline 46 & -10 & -13.0 & 150 & 52 & 0.72 & Mod1 & Higher ND SLD \\
\hline 47 & -10 & -13.0 & 150 & 36.7 & 0.65 & Mod1 & Big dif in Uniformity? \\
\hline 48 & -10 & -13.0 & 150 & 15.3 & 0.43 & Mod1 & 15 um Mod1 Baseline \\
\hline 49 & -10 & -13.0 & 150 & 40.9 & 1.19 & Mod1 & 40 um Mod1 Baseline \\
\hline 50 & -10 & -13.0 & 150 & 14.9 & 0.81 & Std & 15 um Std Baseline \\
\hline 51 & -10 & -13.0 & 150 & 41.4 & 1.83 & Std & 40 um Std Baseline \\
\hline 52 & -10 & -13.0 & 150 & 266 & 0.92 & Mod1 & Largest Cal'd SLD \\
\hline 53 & -10 & -13.0 & 150 & 460 & 1.26 & Mod1 & Max SLD \\
\hline 56 & -10 & -13.0 & 150 & 235 & 0.82 & Mod1 & SLD Uniformity Ref \\
\hline 59 & -10 & -13.0 & 150 & 235 & 0.82 & Mod1 & SLD Uniformity Ref \\
\hline 60 & -10 & -13.0 & 150 & 309 & 1.26 & Mod1 & Max SLD \\
\hline \multicolumn{8}{|c|}{ Repeat Baseline Grid Sprays } \\
\hline 54 & -10 & -13.0 & 150 & 21.5 & 0.70 & Mod1 & Baseline Mod1 \\
\hline 55 & -10 & -13.0 & 150 & 21.4 & 1.39 & Std & \multirow[t]{2}{*}{ Baseline Std } \\
\hline \multicolumn{7}{|c|}{ Distortion Checks } & \\
\hline 57 & -10 & -13.0 & 150 & 19.7 & 2.53 & Std & Bar 3 only (lower, hi LWC) \\
\hline 58 & -10 & -13.0 & 150 & 18.0 & 1.05 & Std & Bar 3 only (lower) \\
\hline 61 & -10 & -13.0 & 150 & 18.0 & 1.05 & Std & Col 18 only (S side) \\
\hline 62 & -10 & -13.0 & 150 & 18.0 & 1.05 & Std & Col 42 only ( $\mathrm{N}$ side) \\
\hline 63 & -10 & -13.0 & 150 & 18.0 & 1.05 & Std & Bar 8 only (upper) \\
\hline 64 & -10 & -13.0 & 150 & 18.0 & 1.05 & Std & Bar 6 only (center) \\
\hline 66 & -10 & -13.0 & 150 & 18.0 & 1.05 & Std & Col 26 only (center) \\
\hline \multicolumn{8}{|c|}{ Repeat Baseline Grid Sprays } \\
\hline 67 & -10 & -13.0 & 150 & 21.5 & 0.70 & Mod1 & Baseline Mod1 \\
\hline 68 & -10 & -13.0 & 150 & 21.4 & 1.39 & Std & Baseline Std \\
\hline \multicolumn{8}{|c|}{ Low Speed Study } \\
\hline 69 & -10 & -11.3 & 100 & 21.5 & 0.93 & Mod1 & Baseline Mod1 \\
\hline 70 & -10 & -11.3 & 100 & 21.4 & 1.83 & Std & Baseline Std \\
\hline 71 & -10 & -10.3 & 50 & 21.6 & 0.94 & Mod1 & Low Spd Mod1, Pair $=10$ \\
\hline 72 & -10 & -10.3 & 50 & 21.5 & 1.62 & Mod1 & Low Spd Mod1, Pair $=20$ \\
\hline 73 & -10 & -10.3 & 50 & 21.1 & 2.07 & Mod1 & Low Spd Mod1, Pair = 30 \\
\hline \multicolumn{8}{|c|}{ Repeat Baseline Grid Sprays } \\
\hline 74 & -10 & -13.0 & 150 & 21.5 & 0.70 & Mod1 & Baseline Mod1 \\
\hline 75 & -10 & -13.0 & 150 & 21.4 & 1.39 & Std & \multirow[t]{2}{*}{ Baseline Std } \\
\hline \multicolumn{4}{|c|}{ Repeat Bonus Baseline } & & & & \\
\hline 76 & -10 & -13.0 & 150 & 15.3 & 0.43 & Mod1 & 15 um Mod1 Baseline \\
\hline 77 & -10 & -13.0 & 150 & 40.9 & 1.19 & Mod1 & 40 um Mod1 Baseline \\
\hline 78 & -10 & -13.0 & 150 & 14.9 & 0.81 & Std & 15 um Std Baseline \\
\hline 79 & -10 & -13.0 & 150 & 41.4 & 1.83 & Std & 40 um Std Baseline \\
\hline
\end{tabular}

TTTSC: Temperature Total, Test Section, Celsius TSTSC: Temperature Static, Test Section, Celsius UTSK: Axial Velocity, Test Section, Knots

MVD: $\quad$ Median Volumetric Diameter, $\mu m$

LWC: Liquid Water Content, $\mathrm{g} / \mathrm{m}^{3}$

American Institute of Aeronautics and Astronautics 